\title{
An Upper Mass Limit on a Red Supergiant Progenitor for the Type II-Plateau Supernova SN 2006my ${ }^{1}$
}

\author{
Douglas C. Leonard, ${ }^{2}$ Avishay Gal-Yam, ${ }^{3}$ Derek B. Fox, ${ }^{4}$ P. B. Cameron, ${ }^{5}$ Erik M. Johansson, ${ }^{6}$ \\ Adam L. Kraus, ${ }^{5}$ David Le Mignant, ${ }^{6}$ And Marcos A. van Dam, ${ }^{6}$ \\ Received 2008 September 12; accepted 2008 October 20; published 2008 November 20
}

\begin{abstract}
We analyze two pre-supernova (SN) and three post-SN high-resolution images of the site of the Type II-Plateau supernova SN 2006my in an effort to either detect the progenitor star or to constrain its properties. Following image registration, we find that an isolated stellar object is not detected at the location of SN 2006my in either of the two pre-SN images. In the first, an $I$-band image obtained with the Wide-Field and Planetary Camera 2 on board the Hubble Space Telescope, the offset between the SN 2006my location and a detected source ("Source 1") is too large: $\geq 0.08$ ", which corresponds to a confidence level of non-association of $96 \%$ from our most liberal estimates of the transformation and measurement uncertainties. In the second, a similarly obtained $V$-band image, a source is detected ("Source 2") that has overlap with the SN 2006my location but is definitively an extended object. Through artificial star tests carried out on the precise location of SN 2006my in the images, we derive a $3 \sigma$ upper bound on the luminosity of a red supergiant that could have remained undetected in our pre-SN images of $\log L / L_{\odot}=5.10$, which translates to an upper bound on such a star's initial mass of $15 M_{\odot}$ from the STARS stellar evolutionary models. Although considered unlikely, we can not rule out the possibility that part of the light comprising Source 1, which exhibits a slight extension relative to other point sources in the image, or part of the light contributing to the extended Source 2, may be due to the progenitor of SN 2006my. Only additional, highresolution observations of the site taken after SN 2006my has faded beyond detection can confirm or reject these possibilities.
\end{abstract}

\section{INTRODUCTION}

The most common class of core-collapse supernovae ( $\mathrm{SNe}$ ) displays a distinct plateau in its optical light curve and is therefore dubbed Type II-Plateau (II-P; see Filippenko 1997 for a review of SN classifications). This type of stellar explosion has long been thought to result from the core collapse and subsequent envelope ejection of isolated red supergiant (RSG) stars, but it is only in recent years that direct observational

\footnotetext{
${ }^{1}$ Some of the data presented herein were obtained at the W. M. Keck Observatory, which is operated as a scientific partnership among the California Institute of Technology, the University of California, and the National Aeronautics and Space Administration. The Observatory was made possible by the generous financial support of the W. M. Keck Foundation. Additional data were obtained from the data archive at the Space Telescope Science Institute. STScI is operated by the Association of Universities for Research in Astronomy, Inc., under NASA contract NAS 5-26555.

${ }^{2}$ Department of Astronomy, San Diego State University, San Diego, CA; leonard@sciences.sdsu.edu

${ }^{3}$ Benoziyo Center for Astrophysics, Weizmann Institute of Science, 76100 Rehovot, Israel

${ }^{4}$ Department of Astronomy and Astrophysics, Pennsylvania State University, 525 Davey Laboratory, University Park, PA

${ }^{5}$ Department of Astronomy, MS 105-24, California Institute of Technology, Pasadena, CA

${ }^{6}$ W. M. Keck Observatory, 65-1120 Mamalahoa Highway, Kamuela, HI
}

evidence of the progenitor-SN II-P connection has begun to accumulate (Van Dyk et al. 2003; Smartt et al. 2004; Maund et al. 2005; Li et al. 2006; Eldridge et al. 2007; Schawinski et al. 2008).

By registering pre-SN and post-SN images, usually taken at high resolution using either space-based optical detectors or ground-based infrared detectors equipped with laser guide star adaptive optics systems, progenitor star identifications have now been proposed for seven SNe II-P (for a contemporary review, see Smartt et al. 2008, and references therein). Although different in detail, all seven proposed progenitors have properties consistent with those of supergiant stars. Because the field is still in its infancy - at this point none of the proposed progenitor objects has been definitively confirmed by its absence in highresolution images of the SN site taken after the SN has faded beyond detection-it is imperative to carefully examine every new progenitor claim. Here we investigate the status of the progenitor of SN 2006my, for which Li et al. (2007; hereafter L07) recently proposed the identification of a RSG progenitor in pre$\mathrm{SN}$ images and derived a zero-age main-sequence mass for it of $M_{\text {ZAMS }}=10_{-3}^{+5} M_{\odot}$.

Although discovered several months after explosion (Stanishev \& Nielsen 2005), the photometry and spectroscopy of SN 2006my presented by L07 clearly establish it as an SN II-P. To identify the progenitor star, L07 register post-SN 
ground-based optical images taken with the Canada-FranceHawaii Telescope in the Sloan $r^{\prime}$ band under excellent seeing conditions (typical stellar full-width at half-maximum $\left.[\mathrm{FWHM}]=0.6^{\prime \prime}\right)$ with pre-SN Hubble Space Telescope (HST) Wide-Field and Planetary Camera 2 (WFPC2) images (typical stellar FWHM $=0.15^{\prime \prime}$ on the WF2 chip, on which the SN site is located), and identify a source in the pre-SN images within the $1 \sigma$ error circle estimated from the transformation uncertainty. Here, we reexamine this identification with the benefit of two new sets of high-resolution, post-SN images: one taken with the wide-field channel of the Near Infrared Camera 2 operated behind the laser guide star assisted adaptiveoptics system (Wizinowich et al. 2006) on the Keck II 10-m telescope (stellar FWHM = 0.10"; note that SN 2006my served as its own tip-tilt star for the observations), and the other taken with the HST WFPC2 camera (with the SN centered on the PC chip; typical stellar FWHM $=0.08^{\prime \prime}$ ) as part of a study of the progenitors of core-collapse supernovae (GO 10803; PI: Smartt).

This paper is organized as follows. In $\S 2.1$ we present the pre-SN and post-SN images and details of the photometric measurements performed on them; in $\S 2.2$ we describe the image registration process along with our conclusion that no isolated stellar source is detected at the location of SN 2006my in either of the pre-SN images; in $\S 2.3$ we estimate detection limits in the pre-SN images; and in $\S 2.4$ we derive an upper mass limit on the progenitor of SN 2006my under the assumption that it was a RSG. We summarize our findings in $\S 3$.

\section{DATA ANALYSIS}

\subsection{Pre-SN Image Photometry with HSTphot}

Table 1 lists information on the five sets of image data considered by our study, hereafter referred to by the designation assigned to the final combined image from each data set. We first discuss our photometry of the two pre-SN images, V1 and I1. The individual frames comprising these datasets were preprocessed through the standard Space Telescope Science Institute pipeline using the latest calibrations as of 2008 July 23. The images were then further processed following the procedure described by Leonard et al. (2003), which employs the suite of programs designed specifically for the reduction of WFPC2 data that are available as part of the HSTphot (Dolphin 2000b) software package (ver. 1.1.7b; our implementation includes the most recent update of 2008 July 19). The hstphot task automatically accounts for WFPC2 point-spread function (PSF) variations, charge-transfer effects across the chips, zeropoints, and aperture corrections.

We performed photometry on the final, combined images using the hstphot task with option flag 10, which combines turning on local sky determination, turning off empirically determined aperture corrections (using default values instead), and turning on PSF residual determination. We ran hstphot on $\mathrm{V} 1$ and I1 individually with a signal-to-noise ratio $(\mathrm{S} / \mathrm{N})$ threshold of 1.0.

In addition to flight-system magnitudes and uncertainties, hstphot returns several measurement parameters for each object detected. For our purposes, the most important of these are the "object type" and "sharpness" parameters. To determine the object type, hstphot compares the goodness of fit (i.e., the $\chi$ value; see Dolphin 2000b) of the detected source's spatial profile with three different models: (1) the best stellar profile (i.e., the library PSF + residuals); (2) a single "hot" pixel without background; and (3) a completely flat profile. If the stellar profile provides the best match, the object is labeled a "star" and designated as object Type 1, 2, or 3, depending on whether it is deemed a "good star," a "possible unresolved binary," or a "bad star" (a star centered on a bad pixel), respectively. If the single "hot" pixel provides the best profile match, the object is labeled a "single-pixel cosmic ray or hot pixel" and designated as object Type 4. Finally, if a flat profile matches best, the object is labeled an "extended object" and designated as object Type 5. Dolphin (2000b) notes that because this is, by design, a conservative test for object type discrimination (i.e., it is a high

TABLE 1

OBSERVATIONS OF NGC 4651

\begin{tabular}{|c|c|c|c|c|c|c|c|c|}
\hline Telescope & Instrument & $\begin{array}{c}\text { Data archive } \\
\text { name }\end{array}$ & UT Date & $\begin{array}{l}\text { Exposure times } \\
\text { (s) }\end{array}$ & Filter & $\begin{array}{c}\text { Plate scale } \\
\left(\operatorname{arcsec} \text { pixel }^{-1}\right)\end{array}$ & $\begin{array}{l}\text { FWHM } \\
(\operatorname{arcsec})\end{array}$ & $\begin{array}{c}\text { Combined image } \\
\text { designation }\end{array}$ \\
\hline$H S T$ & WFPC2 & u2dt0901/2/3t & 1994 May 20 & $60+300+300$ & F555W & 0.10 & 0.15 & V1 \\
\hline HST & WFPC2 & u2dt0904/5/6t & 1994 May 20 & $60+300+300$ & F814W & 0.10 & 0.15 & $\mathrm{I} 1$ \\
\hline Keck II $\ldots .$. & LGS/NIRC2 & $\ldots$ & 2006 Nov 28 & $10 \times 6 \times 10$ & $\mathrm{~K}_{p}$ & 0.04 & 0.10 & K1 \\
\hline$H S T$ & WFPC2 & u9ox0301/2/3/4m & 2007 Apr $26 / 27$ & $4 \times 300$ & $\mathrm{~F} 555 \mathrm{~W}$ & 0.05 & 0.08 & V2 \\
\hline$H S T$ & WFPC2 & u0ox0305/6m & 2007 Apr 27 & $500+700$ & F814W & 0.05 & 0.08 & $\mathrm{I} 2$ \\
\hline
\end{tabular}

NOTES.-SN 2006my in NGC 4651 was discovered in 2006 November 8.82 UT (Nakano \& Itagaki 2006). Pre-explosion HST/WFPC2 images (V1 and I1) were obtained as part of a kinematic study of the core of NGC 4651 (GO 5375; PI: Rubin). Post-explosion image K1 was reduced according to the methods detailed by Gal-Yam et al. (2005). Details on the reduction and analysis of all images obtained with HST/WFPC2 are given in the text. Plate scale is pixel size of the detector chip on which the site of SN 2006my is located (i.e., WF2 for V1 and I1, PC for V2 and I2, and wide-field channel for K1), and FWHM is the measured full width at half-maximum of a point source in each final, combined image. We note that the scale of the wide camera $\left(40^{\prime \prime} \times 40^{\prime \prime}\right)$ is larger than the isoplanatic patch size, which yields a variable PSF shape over the full image. 
threshold to be classified as anything other than a good star), many nonstellar objects will still be classified as Type 1 objects. For particular objects of interest, therefore, examination of the "sharpness" parameter is recommended, where sharpness values between -0.3 (object PSF broader than library PSF) and +0.3 (object PSF narrower than library PSF) indicate confident pointsource detections (Dolphin 2000b).

Figures $1 a$ and $1 b$ show an $\sim 1^{\prime \prime}$ region surrounding the site of SN 2006my on pre-SN images I1 and V1 (respectively), following transformation of both images to the pixel grid of image $\mathrm{K} 1$ (see $\S 2.2$ ). The SN 2006my site is rather complex, and while common objects are evident in Figures $1 a$ and $1 b$, the immediate vicinity of SN 2006my appears quite different in them. In I1, an object of Type 1 ("good star") is reported by hstphot close to the SN location with a $\mathrm{S} / \mathrm{N}$ of 5.6; we label this object "Source 1" in Figure 1a. In V1, an object of Type 5 ("extended object") is detected by hstphot with a $\mathrm{S} / \mathrm{N}$ of 4.70 ; this extended source appears to occupy a "horseshoe-shaped" region that includes the SN location. We label this object "Source 2" in Figure 1b. These two sources appear to be distinct from one another in the combined images.

Source 1 is the object identified by L07 as the probable progenitor of SN 2006my. We confirm both the pixel location and photometry reported by L07 (also determined using HSTphot), with values measured by us (L07) of $[x, y]=[410.23,158.59]$ $([410.22,158.63]))^{7}$ and flight-system magnitude $\mathrm{F} 814 \mathrm{~W}=24$ $.48 \pm 0.19 \mathrm{mag}$ ( $24.47 \pm 0.20 \mathrm{mag}$ ), which corresponds to $I=$ $24.46 \pm 0.19 \mathrm{mag}(24.45 \pm 0.20 \mathrm{mag})$ following transformation according to the prescriptions of Holtzman et al. (1995) and Dolphin (2000a). As noted by L07, although formally classified as an object of Type 1 ("good star") by hstphot, Source 1 exhibits a slight east-west extension in the original WFPC2 image (see Fig. 1a; see also Figure 6 of L07). While L07 conclude that this object is most likely a single star, we note that hstphot reports a "sharpness" value of -0.35 for it, which places it beyond the limits suggested by Dolphin (2000b) for confident point-source detections. Source 2 is unequivocally an extended source, as hstphot flags it as an object of Type 5 and measures its profile to have a sharpness of -0.58 , well beyond the range for isolated starlike sources and for which hstphot's PSF star-fitting routines provide reliable photometry (Dolphin \& Kennicutt 2002). We note that this object may be the "extended source" mentioned by L07 but not further investigated due to its location near the $\sim 2 \sigma$ error radius of their derived SN pixel coordinates.

\footnotetext{
${ }^{7}$ Note that all pixel coordinates given in this paper are in the "IRAF" systemthe coordinate system reported by using the imexamine task within IRAF or by running DAOPHOT (Stetson 1987) — in which an integer value is assigned to a star that is centered on the center of a pixel. These pixel values are 0.5 greater in both $x$ and $y$ than those reported by hstphot, which follows the DoPHOT (Schechter et al. 1993) convention of assigning an integer value to a star that is centered in the lower left corner of a pixel.
}

We shall return to further discuss both of these "sources of interest" following a description of the image registration process.

\subsection{Image Registration}

To determine whether the progenitor star that exploded as SN 2006my is detected in the pre-SN images, we first registered image I1 to image $\mathrm{K} 1$ by using the IRAF tasks geomap and geotran, closely following the technique described by Gal-Yam et al. (2005). To carry out the transformation, we used 16 common sources and obtained a final solution with a rms residual of 0.30 pixel in $x$ and 0.27 pixel in $y$, in the $\mathrm{K} 1$ pixel grid. We then similarly registered images $\mathrm{V} 1, \mathrm{~V} 2$, and $\mathrm{I} 2$ to the transformed I1 image (using more than a dozen common sources in each case), which resulted in five final images all registered to the common $\mathrm{K} 1$ pixel grid.

Using the transformed images, we then measured the pixel locations of Source 1 (in image I1) and SN 2006my (in images $\mathrm{K} 1, \mathrm{I} 2$, and $\mathrm{V} 2$ ) using the centroiding algorithm of the imexamine task within IRAF. ${ }^{8}$ The results returned by imexamine depend somewhat on both the centering radius and fitting function employed. Thus, we applied a range of values - centering radii of from 3-10 pixels, inclusive, using both Gaussian and Moffat profiles - and took the average as our "best" value and the measured scatter around the average as the measurement uncertainty. Due to the extended nature of Source 2 in image V1, it was not possible to estimate a precise pixel location for it by using the IRAF centroiding algorithm; instead, we ran the source-finding program SExtractor (Bertin \& Arnouts 1996) on the image, which reported a nonstellar source and pixel position at the location of Source 2. The pixel locations, measurement uncertainties, and transformation uncertainties of all objects are given in Table 2.

Accounting for measurement and transformation uncertainties, we determine offsets between Source 1 in image I1 and SN 2006my in images K1, I2, and V2, of $0.083^{\prime \prime} \pm 0.017^{\prime \prime}$, $0.080^{\prime \prime} \pm 0.016^{\prime \prime}$, and $0.086^{\prime \prime} \pm 0.016^{\prime \prime}$, which correspond to separations significant at the $4.8 \sigma, 4.9 \sigma$, and $5.2 \sigma$ levels, respectively, for a two-dimensional (i.e., both $x$ and $y$ ) Gaussian. This indicates non-association at greater than the $99 \%$ confidence level (see the Appendix for a full discussion of how significance of source separation is determined), implying that the object detected as Source 1 is in all likelihood not the progenitor of SN 2006my. Due to the extended nature of Source 2, it is not possible to derive a similarly well-quantified offset and uncertainty estimate between it and SN 2006my, but we note that from the location reported by SExtractor, its formal separation is only $0.024^{\prime \prime}$, and examination of Figure 1 reveals that the location of SN 2006my is indeed coincident with part of the extended region identified as Source 2.

\footnotetext{
${ }^{8}$ IRAF is distributed by the National Optical Astronomy Observatories, which are operated by the Association of Universities for Research in Astronomy, Inc., under cooperative agreement with the National Science Foundation.
} 


\section{LEONARD ET AL.}

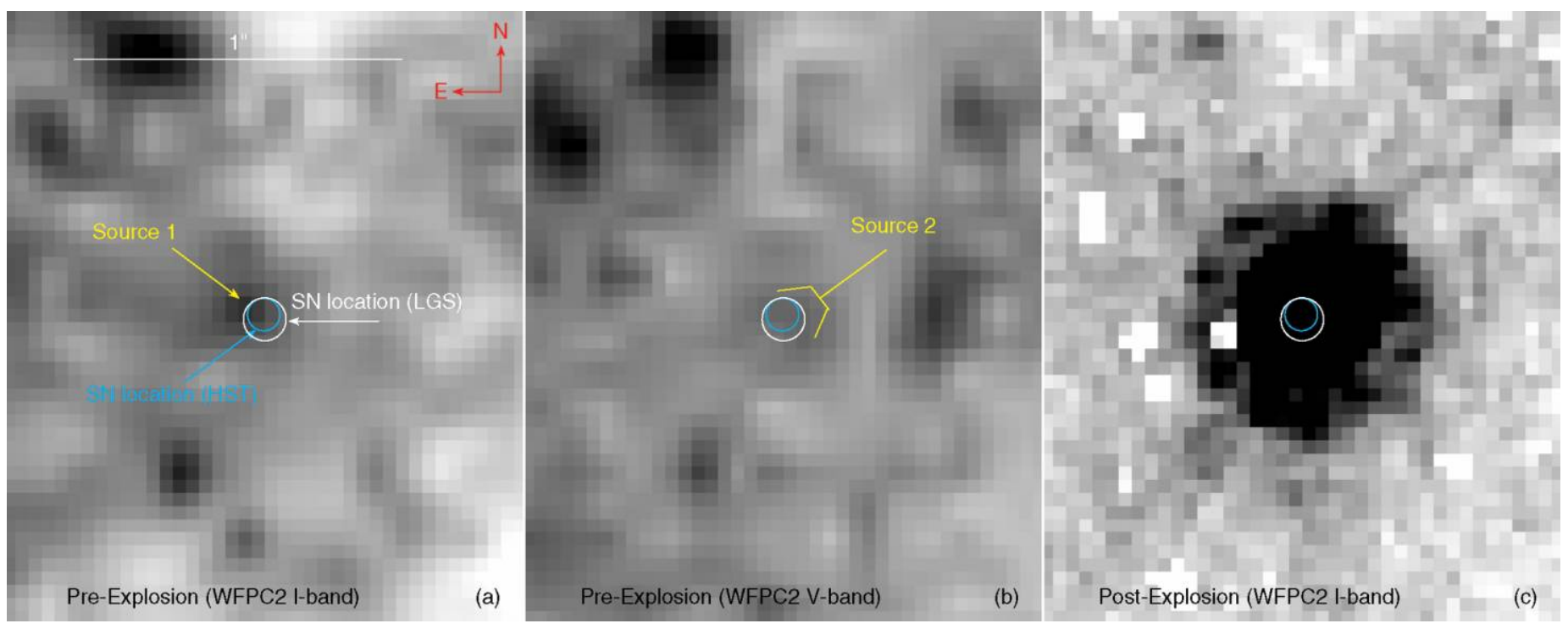

FIG. 1.-Site of SN 2006my in pre-SN images I1 (panel $a$; all image designations are as given in Table 1) and V1 (panel $b$ ), and post-SN image I2 (panel $c$ ). All images have been transformed and resampled to the pixel grid of the K1 image ( $\$ 2.2)$. The cyan circles indicate the approximate $5 \sigma$ uncertainty (for a two-dimensional Gaussian; see the Appendix) of the position of SN 2006my relative to the transformed I1 image as measured in the transformed I2 image. The white circles indicate the same level of uncertainty in the location of SN 2006my as measured in the K1 image relative to the transformed I1 image. Two "sources of interest," discussed in the text, are labeled Source 1 and Source 2 in panels $a$ and $b$, respectively.

To serve as a check on the results obtained by transforming all images to the K1 frame, we also performed a direct, "HSTonly," registration between images V2 and V1, and I2 and I1, using the hstphot-reported pixel positions (with an additional correction for distortion using the solutions of Anderson \& King 2003) for all $(>20)$ objects used to determine the transformation. Results of these registrations are given in Table 3. To assign a measurement uncertainty on the hstphot-measured object pixel positions, we consulted Figure 4 of Dolphin (2000b), which presents estimates of the $1 \sigma$ astrometry error for sources detected by hstphot as a function of count level. For the count level of Source $1(\sim 45)$, a total astrometry error estimate of $\sim 0.4 \mathrm{WF} 2$ pixel, or $\sim 0.29$ pixels in both $x$ and $y$, is determined, while for SN 2006my (counts $>20,000$ ) position uncertainties of only 0.03 pixels in $x$ and $y$ are derived.

Accounting for measurement and transformation uncertainties, we determine the separation between Source 1 in image I1 in the transformed location of SN 2006my from image I2 to be $0.082^{\prime \prime} \pm 0.031^{\prime \prime}$, which represents an offset of $2.6 \sigma$ for a twodimensional Gaussian, indicating non-association at the $96 \%$ confidence level (see the Appendix). This separation is nearly identical to those determined in the K1 pixel frame, although the

TABLE 2

Image Transformations to LGS (Image K1) Pixel Grid

\begin{tabular}{|c|c|c|c|c|c|c|c|}
\hline \multirow[b]{2}{*}{ IMAGE } & \multirow[b]{2}{*}{ ОВЈЕСт } & \multirow[b]{2}{*}{$\mathrm{X}$} & \multirow[b]{2}{*}{$\mathrm{Y}$} & \multicolumn{2}{|c|}{ MEASUREMENT UNCERTAINTY } & \multicolumn{2}{|c|}{ TRANSFORMATION UNCERTAINTY } \\
\hline & & & & $\sigma_{x}$ & $\sigma_{y}$ & $\sigma_{x}$ & $\sigma_{y}$ \\
\hline$\ldots$ & Source 1 & 579.55 & 543.28 & 0.34 & 0.04 & $\ldots$ & $\ldots$ \\
\hline $\mathrm{K} 1 \ldots$. & SN 2006my & 581.55 & 542.72 & 0.05 & 0.03 & 0.30 & 0.27 \\
\hline $\mathrm{I} 2 \ldots \ldots$ & SN 2006my & 581.47 & 543.07 & 0.05 & 0.02 & 0.21 & 0.22 \\
\hline $\mathrm{V} 2 \ldots \ldots$ & SN 2006my & 581.69 & 543.07 & 0.02 & 0.02 & 0.23 & 0.25 \\
\hline $\mathrm{V} 1 \ldots$. & Source 2 & 581.95 & 542.55 & $\ldots$ & $\ldots$ & 0.15 & 0.15 \\
\hline
\end{tabular}

NOTES.-Location of sources identified in Fig. 1 in images listed in Table 1, following transformation of all images to the K1 pixel grid. All transformation uncertainties are given in pixel units (with a plate scale of $0.04^{\prime \prime}$ pixel $^{-1}$; see Table 1) and represent the rms residuals following registration of each image to image I1 transformed onto the K1 grid. Because Source 2 is extended (and not symmetric), we do not assign a measurement uncertainty on its position. Measurement uncertainties were derived as discussed in the text $(\$ 2.2)$. Note the particularly large uncertainty in the $x$ coordinate of Source 1 in transformed image I1, likely due to the slight extension of the object along the $x$ (i.e., eastwest) direction, as discussed in the text (\$ 2.2) and evident in Fig. $1 a$. 
TABLE 3

Image Transformations to 1994 WFPC2 (IMAges V1 AND I1) PiXel Grid

\begin{tabular}{|c|c|c|c|c|c|c|c|}
\hline \multirow[b]{2}{*}{ IMAGE } & \multirow[b]{2}{*}{ ОВJECT } & \multirow[b]{2}{*}{$\mathrm{X}$} & \multirow[b]{2}{*}{$\mathrm{Y}$} & \multicolumn{2}{|c|}{ MEASUREMENT UNCERTAINTY } & \multicolumn{2}{|c|}{ TRANSFORMATION UNCERTAINTY } \\
\hline & & & & $\sigma_{x}$ & $\sigma_{y}$ & $\sigma_{x}$ & $\sigma_{y}$ \\
\hline$\ldots \ldots$ & Source 1 & 410.23 & 158.59 & 0.29 & 0.29 & $\ldots$ & $\ldots$ \\
\hline V1 ..... & Source 2 & 410.31 & 159.58 & $\ldots$ & $\ldots$ & $\ldots$ & $\ldots$ \\
\hline $\mathrm{I} 2 \ldots \ldots$ & SN 2006my & 410.18 & 159.41 & 0.03 & 0.03 & 0.15 & 0.12 \\
\hline $\mathrm{V} 2 \ldots$. & SN 2006my & 410.20 & 159.51 & 0.03 & 0.03 & 0.12 & 0.09 \\
\hline
\end{tabular}

Notes.-Location of sources identified in Fig. 1 in images defined in Table 1. Transformation uncertainties are given in pixel units (with a plate scale of $0.10 \mathrm{pixel}^{-1}$; see Table 1) and represent the rms residuals following registration of I2 onto I1 and V2 onto V1. The locations of Source 1 and Source 2 in images I1 and V1, respectively, are those reported by hstphot. The locations of SN 2006my in the V1 and I1 pixel grids were derived by taking the hstphot-reported coordinates of SN 2006my in images V2 and I2 and transforming them using the geoxytran task in IRAF to the V1 and I1 pixel grids, respectively. Measurement uncertainties for all object locations are those suggested by Dolphin (2000b). Because Source 2 is extended (and not symmetric), we do not assign a measurement uncertainty on its position.

significance of the offset is lower. This is due mainly to the larger uncertainty adopted for the pixel location of Source 1 in I1 than was found empirically for the I1 image transformed to the K1 pixel grid. Registration of I1 onto K1 resamples the data onto a finer grid and therefore likely provides a better localization of Source 1, although, as noted by L07, resampling the data has the risk of smoothing several (extended) sources into a point source. We also note that a direct registration of V2 to I1 (not listed in Table 3) yields an offset between Source 1 and SN 2006my of $0.092^{\prime \prime} \pm 0.032^{\prime \prime}$, which represents an offset of $2.9 \sigma$ for a two-dimensional Gaussian and indicates nonassociation at the $98 \%$ confidence level. With all of these lines of evidence, we conclude that a $96 \%$ confidence level for nonassociation is a conservative estimate of the significance of the offset between Source 1 and SN 2006my.

We note that in the original I1 WFPC2 image, Source 1 presents itself as elevated flux in two pixels (i.e., pixels [411, 158] and [410, 159]; see Figure 6 of L07), one of which contains our derived location of SN 2006my at its far edge (Table 3). The slight extension of Source 1 noted earlier (§ 2.1) raises the possibility that this source may, in fact, contain light from more than one object-for instance, two RSG lying in adjacent WF pixels, or perhaps a compact star cluster —an idea considered by L07 but deemed improbable. This lingering possibility can only be definitively removed from consideration by future high-resolution imaging after the SN has faded beyond detection. For now, we conclude that from our astrometric measurements, an isolated stellar object is not detected at the location of SN 2006my in either of the two preSN images: In image I1, the offset between the SN 2006my location and the source detected by hstphot is too large, and in V1 the source that overlaps with the SN 2006my site is extended. The relevant question that arises, then, is this: How faint a point source at the precise location of SN 2006my would we have confidently detected as a point source in the pre-SN images?

\subsection{Detection Limits in Pre-SN Images}

To set accurate detection limits on a point source at the location of SN 2006my in the pre-SN images, we proceeded as follows. First, we used the showpsf task within HSTphot to produce the library PSFs appropriate for point sources at the precise pixel locations (accurate to 0.1 pixel) of SN 2006my in the V1 and I1 images. Using these PSFs, we then inserted artificial stars of known flux (corresponding to $21.5<V<27.0$ and $20.5<I<26.0)$ at the SN locations in the V1 and I1 images. Then, we ran hstphot on these images in exactly the same manner as we did when seeking a progenitor star in the original images.

To be considered a confident "detection" of a single star at the SN 2006my location, we demanded that the object be classified by hstphot as a "good star" (Type 1), have a sharpness between -0.3 and +0.3 , and have a reported pixel location no more than $1 \sigma$ away from the nominal SN location, where the uncertainty accounts for the measurement and transformation uncertainties listed in Table 3 as well as the astrometric uncertainty appropriate for the object from Figure 4 of Dolphin (2000b).

From this analysis, we derive detection limits of $V=24.9 \pm$ $0.3 \mathrm{mag}$ and $I=24.4 \pm 0.2 \mathrm{mag}$ for point sources at the location of SN 2006my in the V1 and I1 images, respectively. In both cases the limiting magnitude is set by the sharpness parameter becoming less than -0.3 (i.e., source too extended to be confidently considered a point source). We note that these detection limits are significantly shallower than those derived by L07, in which detection limits were derived by examining the magnitudes of all $3 \sigma$ detections in the images. Because we are specifically interested in the point-source detection limits at the location of the SN, we consider our (less restrictive) limits to more accurately reflect the relevant detection threshold.

\subsection{Properties of the Progenitor Star}

To convert our detection thresholds to constraints on the initial mass of a RSG progenitor star that could have exploded 
as SN 2006my, we employ the metallicity-dependent stellar models produced with the Cambridge stellar evolution code, STARS, the descendant of the code developed originally by Eggleton (1971) and updated most recently by Eldridge \& Tout (2004; see Smartt et al. 2008 and references therein). ${ }^{9}$ The models follow stellar evolution up to the initiation of core neon burning, which is likely to give an accurate indication of the preSN luminosity; comparisons with other contemporary model grids (i.e., Heger \& Langer 2000; Meynet \& Maeder 2000) show that the endpoints for stars in the $8 \rightarrow 15 \mathrm{M}_{\odot}$ range differ by at most 0.2 dex in luminosity among the codes (Smartt et al. 2004).

Using the metallicity and radial gradient in NGC 4651 published by Pilyugin et al. (2004), L07 derive a metallicity at the SN 2006my location of $\log (O / H)+12=8.51 \pm 0.06$, which is subsolar according to the recent analysis of Asplund et al. (2004), who found $[O / H]=8.66 \pm 0.05$. We thus use the $Z=$ 0.01 (the closest metallicity calculated) STARS stellar evolution models as the basis for deriving the upper mass limit for our study. Figure 2 displays the final predicted luminosity for stars with masses between 8 and $20 M_{\odot}$ from these models.

We now seek to determine the greatest possible luminosity that a RSG could have had and still have remained undetected by our analysis of the pre-SN images. Since it will prove to be far more restrictive, we begin by considering the I1 image, and calculate the bolometric magnitude of RSG stars corresponding to our detection limit through the equation:

$$
M_{\mathrm{bol}}=-\mu-A_{V}+I+(V-I)_{\mathrm{RSG}}+\mathrm{BC}_{V},
$$

where $\mu$ is the distance modulus of NGC $4651, A_{V}$ the extinction to SN 2006my, $I$ the $I$-band detection threshold, $(V-I)_{\mathrm{RSG}}$ the color range of RSG stars (i.e., spectral types K3 $\rightarrow$ M4), and $\mathrm{BC}_{V}$ the bolometric correction corresponding to each $(V-I)_{\mathrm{RSG}}$. As detailed by $\mathrm{L} 07$, distance estimates to NGC 4651 span quite a wide range. Because it is our goal here to set the most conservative lower bound on our detection threshold, we adopt the long distance estimate, $\mu=31.74 \pm 0.25 \mathrm{mag}$, found by Solanes et al. (2002) by averaging seven different Tully-Fisher distances to the galaxy. For the extinction, we adopt $A_{V}=0.08 \pm 0.02 \mathrm{mag}$, which represents the Galactic value along the line of sight, because there is no evidence for host-galaxy extinction (L07). For the color and bolometric corrections appropriate for RSG stars, we consult the values reported by Elias et al. (1985), who find that the quantity $\left[(V-I)_{\mathrm{RSG}}+\mathrm{BC}_{V}\right]$ lies in the remarkably tight range $0.88 \rightarrow$ 1.0 for supergiant stars of spectral types K3-M4 (i.e., RSG). Again, because we wish to set the most conservative lower detection limit, we assign $(V-I)_{\mathrm{RSG}}+\mathrm{BC}_{V}=0.88$, which is

\footnotetext{
${ }^{9}$ The models were downloaded from the code's Web site, at http://www.ast .cam.ac.uk/ stars.
}

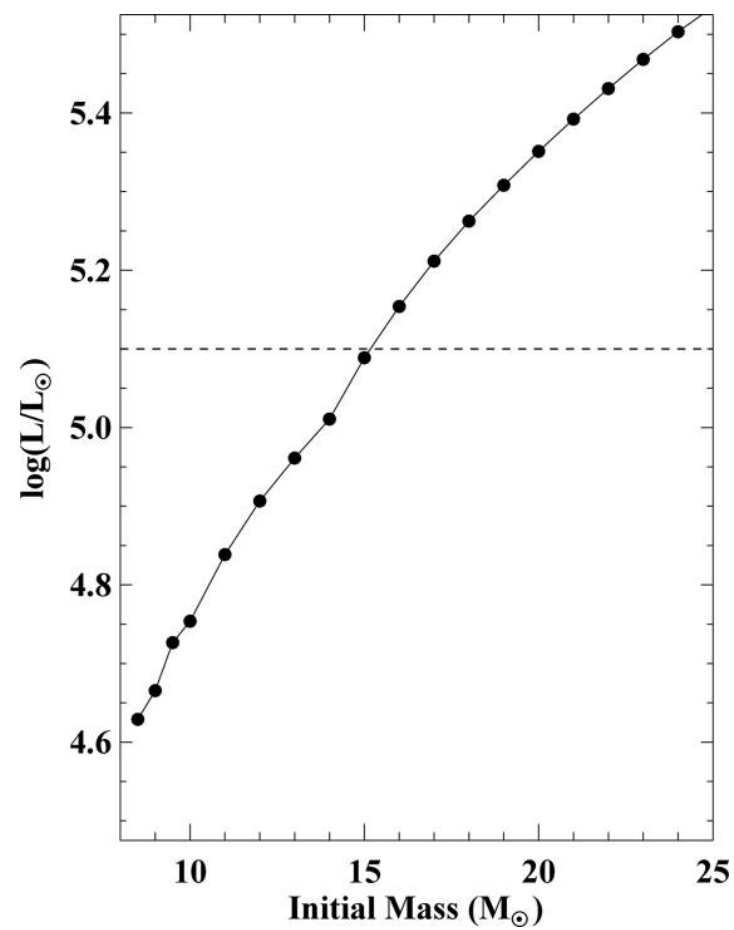

FIG. 2.-Initial mass vs. final predicted luminosity prior to explosion for $Z=$ 0.01 stars evolved with the STARS stellar evolution code (Eldridge \& Tout 2004). The dashed line indicates the $3 \sigma$ upper luminosity limit for a RSG that could have remained undetected by our analysis of pre-SN images of the site of SN 2006my.

the value obtained for an M4 supergiant star. Finally, we set $I=24.4 \pm 0.2 \mathrm{mag}$, as derived in $\S 2.3$.

With these values, we derive $M_{\text {bol }}=-6.54 \pm 0.32 \mathrm{mag}$ as the limiting bolometric magnitude, above which any RSG would have been detected in our pre-SN image. This corresponds to a $3 \sigma$ detection threshold of $M_{\mathrm{bol}}=-7.50 \mathrm{mag}$, which translates to $\log L / L_{\odot}=4.90$. If we adopt a maximum systematic uncertainty of 0.2 dex in the theoretical stellar model endpoints, then the final $3 \sigma$ lower bound on the luminosity of a RSG that would have been confidently detected in our pre-SN image is $\log L / L_{\odot}=5.10$. From Figure 2 this corresponds to an upper bound on the progenitor mass of $M_{\text {ZAMS }}=15 M_{\odot}$, and we therefore conclude that any RSG progenitor with an initial mass greater than this value would have been detected using our analysis procedure.

Applying a similar analysis to the V1 image results in a detection threshold of $\log L / L_{\odot}=6.30$, which unfortunately does not rule out any progenitors up to $200 M_{\odot}$, the highest progenitor mass considered by the STARS models. Our most restrictive limit thus comes from the I1 image.

\section{CONCLUSIONS}

We analyze two pre-SN and three new post-SN high-resolution images of the site of the Type II-Plateau supernova SN 2006my 
in an effort to either detect the progenitor star or to constrain its properties. Our primary result is that we do not detect an isolated stellar object at the location of SN 2006my in either of the two pre-SN images. From our image registration, we therefore do not confirm the association found by L07 between a stellar source (Source 1) in pre-SN $I$-band images (I1) and the location of SN 2006my. Using new high-resolution post-SN images, we derive an offset between SN 2006my and Source 1 of $\geq 0.08^{\prime \prime}$ from the SN location, which represents a confidence level of non-association of more than $96 \%$ from our most liberal estimates of the image transformation and measurement uncertainties. Through artificial star tests carried out at the precise location of SN 2006my in image I1, we derive a $3 \sigma$ upper bound on the mass of the progenitor of SN 2006my of $M_{\text {ZAMS }}$ $=15 M_{\odot}$ from the STARS stellar evolutionary models.

Although considered unlikely, we cannot rule out the possibility that part of the light comprising Source 1, which exhibits some extension relative to other point sources in image I1, or part of the light contributing to Source 2, a definitively extended source detected in pre-SN $V$-band images (V1) that has overlap with the SN 2006my location, may be due to the progenitor of SN 2006my. Only additional, high-resolution observations of the site taken after SN 2006my has faded beyond detection can confirm or reject these possibilities.

We thank an anonymous referee for helpful comments that improved the manuscript. A. G. acknowledges support by the Benoziyo Center for Astrophysics, a research grant from Peter and Patricia Gruber Awards, and the William Z. and Eda Bess Novick New Scientists Fund at the Weizmann Institute. A. L. K. is supported by a NASA Origins grant to L. Hillenbrand. This research has made use of the NASA/IPAC Extragalactic Database (NED), which is operated by the Jet Propulsion Laboratory, California Institute of Technology, under contract with NASA. We wish to recognize and acknowledge the very significant cultural role and reverence that the summit of Mauna Kea has always had within the indigenous Hawaiian community. We are most fortunate to have the opportunity to conduct observations from this mountain.

\section{REFERENCES}

Anderson, J., \& King, I. R. 2003, PASP, 115, 113

Asplund, M., Grevesse, N., Sauval, A. J., Allende Prieto, C., \& Kiselman, D. 2004, A\&A, 417, 751

Bertin, E., \& Arnouts, S. 1996, A\&AS, 117, 393

Dolphin, A. E. 2000a, PASP, 112, 1397

. 2000b, PASP, 112, 1383

Dolphin, A. E., \& Kennicutt, Jr. 2002, AJ, 123, 207

Eggleton, P. P. 1971, MNRAS, 151, 351

Eldridge, J. J., Mattila, S., \& Smartt, S. J. 2007, MNRAS, 376, L52 Eldridge, J. J., \& Tout, C. A. 2004, MNRAS, 348, 201

Elias, J. H., Frogel, J. A., \& Humphreys, R. M. 1985, ApJS, 57, 91

Filippenko, A. V. 1997, ARA\&A, 35, 309

Gal-Yam, A., et al. 2005, ApJ, 630, L29

Heger, A., \& Langer, N. 2000, ApJ, 544, 1016

Holtzman, J. A., Burrows, C. J., Casertano, S., Hester, J. J., Trauger, J. T., Watson, A. M., \& Worthey, G. 1995, PASP, 107, 1065

Leonard, D. C., Kanbur, S. M., Ngeow, C. C., \& Tanvir, N. R. 2003, ApJ, 594, 247

Li, W., Van Dyk, S. D., Filippenko, A. V., Cuillandre, J.-C., Jha, S., Bloom, J. S., Riess, A. G., \& Livio, M. 2006, ApJ, 641, 1060
Li, W., Wang, X., Van Dyk, S. D., Cuillandre, J.-C., Foley, R. J., \& Filippenko, A. V. 2007, ApJ, 661, 1013 (L07)

Maund, J. R., Smartt, S. J., \& Danziger, I. J. 2005, MNRAS, 364, L33

Meynet, G., \& Maeder, A. 2000, A\&A, 361, 101

Nakano, S., \& Itagaki, K. 2006, CBET, 756, 1

Pilyugin, L. S., Vílchez, J. M., \& Contini, T. 2004, A\&A, 425, 849

Schawinski, K., et al. 2008, Science, 321, 223

Schechter, P. L., Mateo, M., \& Saha, A. 1993, PASP, 105, 1342

Smartt, S. J., Eldridge, J. J., Crockett, R. M., \& Maund, J. R. 2008, MNRAS, submitted (ArXiv e-prints 0809.0403)

Smartt, S. J., Maund, J. R., Hendry, M. A., Tout, C. A., Gilmore, G. F., Mattila, S., \& Benn, C. R. 2004, Science, 303, 499

Solanes, J. M., Sanchis, T., Salvador-Solé, E., Giovanelli, R., \& Haynes, M. P. 2002, AJ, 124, 2440

Stanishev, V., \& Nielsen, T. B. 2006, CBET, 737, 1

Stetson, P. B. 1987, PASP, 99, 191

Van Dyk, S. D., Li, W., \& Filippenko, A. V. 2003, PASP, 115, 1289

Wizinowich, P. L., et al. 2006, PASP, 118, 297 


\section{APPENDIX \\ ON THE CONFIDENCE LEVEL OF NON-ASSOCIATION OF POINT SOURCES}

Determining the significance of positional offsets between objects identified in two images is of paramount importance when assessing the potential association between a progenitor star (in a pre-explosion image) and a supernova (in a postexplosion image), where one image has been transformed into the pixel frame of the other. Six sources of uncertainty are typically identified: Positional uncertainty of the putative progenitor star (in both $x$ and $y$ ), positional uncertainty of the SN (in both $x$ and $y$ ), and the uncertainty in the transformation (also in both $x$ and $y$ ). In much of the past work involving SN progenitors (hereafter, the "traditional" approach), offset significance (i.e., how many $\sigma$ away the two objects are) has been derived by taking the measured radial offset between the two objects, and then dividing this value by the quadrature sum of all of the uncertainties. A formal analysis, however, finds this approach to be somewhat in error.

Consider the two-dimensional Gaussian that describes the uncertainty in an SN's measured position $(x, y)$ compared with that of a putative progenitor star $\left(x_{0}, y_{0}\right)$ :

$$
p(x, y) d x d y=\frac{1}{2 \pi \sigma_{x} \sigma_{y}} e^{-\left(x-x_{0}\right)^{2} / 2 \sigma_{x}^{2}} e^{-\left(y-y_{0}\right)^{2} / 2 \sigma_{y}^{2}} d x d y,
$$

where $\sigma_{x(y)}$ represents the quadrature sum of the measurement and transformation errors in $x(y)$. Converting this to a normalized form by defining $\Sigma_{x} \equiv\left(x-x_{0}\right) / \sigma_{x}, \Sigma_{y} \equiv\left(y-y_{0}\right) / \sigma_{y}$ yields:

$p\left(\Sigma_{x}, \Sigma_{y}\right) d \Sigma_{x} d \Sigma_{y}=\frac{1}{2 \pi \sigma_{x} \sigma_{y}} e^{-\left(\Sigma_{x}^{2} / 2\right)} e^{-\left(\Sigma_{y}^{2} / 2\right)}\left(\sigma_{x} d \Sigma_{x}\right)\left(\sigma_{y} d \Sigma_{y}\right)$,

which becomes the expected:

$$
p\left(\Sigma_{x}, \Sigma_{y}\right) d \Sigma_{x} d \Sigma_{y}=\frac{1}{2 \pi} e^{-\left(\Sigma_{x}^{2} / 2\right)} e^{-\left(\Sigma_{y}^{2} / 2\right)} d \Sigma_{x} d \Sigma_{y} .
$$

We convert this normalized Gaussian into radial form with a further change of coordinates $\left(\Sigma_{x} \equiv \rho \cos \theta, \Sigma_{y} \equiv \rho \sin \theta\right)$ and a little algebra to yield:

$$
p(\rho, \theta) d \rho d \theta=\frac{1}{2 \pi} e^{-\rho^{2} / 2} \rho d \rho d \theta
$$

or ignoring the angular part of the distribution,

$$
p(\rho) d \rho=\rho e^{-\rho^{2} / 2} d \rho .
$$

The total integrated probability for $\rho<Q$ (where $Q$ is equal to, e.g., $\left.\left[\Sigma_{x}^{2}+\Sigma_{y}^{2}\right]^{1 / 2}\right)$, then, is

$$
p(\rho<Q)=1-e^{-\left(Q^{2} / 2\right)} .
$$

A simple example serves to highlight the differences between calculating offset significance in the traditional manner versus calculating it according to equation (A1). Suppose a progenitor star candidate and a SN are measured to be located at pixel locations $[10,10]$ and $[11,11]$ on pre-SN and (transformed) postSN images, respectively, with total uncertainties (quadrature sum of all measurement and transformation uncertainties) of $\sigma_{x}=1, \sigma_{y}=1$. In the traditional approach, this would represent an offset of $1.41 \pm 1.41$ pixels, or a separation significant at the $1 \sigma$ level, which implies a confidence level of nonassociation of $68 \%$.

However, if we calculate the total integrated probability for $\rho<1.41$ according to equation (A1), we find $p(\rho<1.41)=1-\exp \left[-\left(1.41^{2} / 2\right)\right]=0.63$, which implies a confidence level of non-association of $63 \%$. Because we are dealing with positions and uncertainties in two dimensions, it thus appears most precise to state the result as follows: "The two objects are located $1.41 \sigma$ away from each other for a two-dimensional Gaussian, which indicates non-association at the $63 \%$ confidence level." We therefore express all of our association significances in this paper in this manner. 